\title{
Homogeneous Stabilizer by State Feedback for Switched Nonlinear Systems Using Multiple Lyapunov Functions’ Approach
}

\author{
Hui Ye, ${ }^{1,2}$ Bin Jiang, ${ }^{1}$ and Hao Yang ${ }^{1}$ \\ ${ }^{1}$ College of Automation Engineering, Nanjing University of Aeronautics \& Astronautics, Nanjing 210016, China \\ ${ }^{2}$ School of Mathematics and Physics, Jiangsu University of Science and Technology, Zhenjiang 212000, China \\ Correspondence should be addressed to Hui Ye; yh781001@163.com
}

Received 20 July 2017; Accepted 31 October 2017; Published 19 November 2017

Academic Editor: Filippo Cacace

Copyright (C) 2017 Hui Ye et al. This is an open access article distributed under the Creative Commons Attribution License, which permits unrestricted use, distribution, and reproduction in any medium, provided the original work is properly cited.

\begin{abstract}
This paper investigates the problem of global stabilization for a class of switched nonlinear systems using multiple Lyapunov functions (MLFs). The restrictions on nonlinearities are neither linear growth condition nor Lipschitz condition with respect to system states. Based on adding a power integrator technique, we design homogeneous state feedback controllers of all subsystems and a switching law to guarantee that the closed-loop system is globally asymptotically stable. Finally, an example is given to illustrate the validity of the proposed control scheme.
\end{abstract}

\section{Introduction}

A switched system is a hybrid system which consists of a family of subsystems, either continuous-time or discrete-time subsystems, and a switching law, which defines a specific subsystem that is active at each instant of time. In the last decade, the problem of designing switching strategies for switched systems has received a great attention $[1-10]$ and the references therein. A switched system may be either stable or unstable, not depending on its subsystems but a particular switching signal. Because of less conservativeness, MLFs method is more preferable than other methods. It plays an important role in design for switched nonlinear systems in the control literature $[4,5]$. Based on the common Lyapunov function approach, the existing designs of switched nonlinear systems were restricted to triangular form under arbitrary switchings via backstepping or forwarding method $[7,8,10]$. For switched nonlinear systems, it has not been used by homogeneous domination control approach to the best of our knowledge. The homogeneous domination approach begins with the linear domination idea proposed in [11], where a global output feedback stabilizer is constructed for uncertain nonlinear system under a linear growth condition. The work [12] deals with the stability of nonswitched nonlinear systems using the homogeneous domination approach. Using such method, how to tackle the problem of stabilization for a class of more general switched nonlinear systems is challenging. In this paper, motivated by [13], in some regular conditions, the MLFs method is utilized to completely handle the different coordinate transformations for all subsystems by constructing proper switching law. Compared with the existing literature on switched nonlinear systems, the results of this paper have some advantages. At first, the problem of global state feedback stabilization for a wider class of nonlinear systems with some special forms is studied by homogeneous domination approach, which simplifies the control design procedure. Secondly, individual coordinate transformations for subsystems are introduced into virtual controllers, which decreases the conservativeness compared with the common change of coordinate. Finally, the dual controllers and a switching law are designed by constructing the MLFs.

The outline of the paper is as follows. In Section 2, we provide the system formulation and preliminaries. In Section 3, we present a necessary and sufficient condition for the stabilization issue and the main result of the paper. An 
illustrative example is given in Section 4. Our conclusion is included in Section 5.

\section{System Description and Preliminaries}

We consider a class of switched nonlinear systems of the form

$$
\begin{aligned}
& \dot{x}_{i}=x_{i+1}+f_{i, \sigma(t)}\left(\bar{x}_{i}\right)+g_{i, \sigma(t)}(x), \quad i=1, \ldots, n-1 \\
& \dot{x}_{n}=u_{\sigma(t)}+f_{n, \sigma(t)}(x),
\end{aligned}
$$

where $x=\left(x_{1}, \ldots, x_{n}\right)^{T} \in \mathbb{R}^{n}$ is the system state and $u_{\sigma(t)}$ is the subsystem input. $\bar{x}_{i}=\left(x_{1}, \ldots, x_{i}\right)^{T}, i=1, \ldots, n$. The function $\sigma(t):[0, \infty) \rightarrow M=\{1, \ldots, m\}$ is a switching signal which is assumed to be a piecewise continuous (from the right) function of time. For each $k \in M, u_{k} \in R$ is the control input of the $k$ th subsystem. For any $k \in M$, and $i=1,2, \ldots, n$, the functions $f_{i, k}(\cdot), i=1, \ldots, n$ and $g_{l, k}(x), l=1, \ldots, n-1, k \in M$ are $C^{\infty}$ with respect to their arguments and vanish at the origin; that is, $f_{i, k}(0)=0$ and $g_{l, k}(0)=0$. In addition, we assume that the state of system (1) does not jump at the switching instants; that is, the solution is everywhere continuous and only finite switches can occur in any finite time interval.

Obviously, the structure of the switched nonlinear system (1) is much more general than the structure of the nonswitched nonlinear systems called strict-feedback form whose stabilization problem has been addressed in $[12,14]$ when $g_{l, k}(x) \equiv 0$ and $m=1$. The existence of a common Lyapunov function for all subsystems was shown to be a necessary and sufficient condition for a switched system to be asymptotically stable under arbitrary switchings [15]. If at least one of the subsystems is not asymptotically stable, we still solve the global stabilization problem for system (1) by designing controllers for all subsystems and a switching law.

In the following, we collect some useful definitions and lemmas which play important roles in this paper. The innovative idea of homogeneity was introduced for the stability analysis of a nonlinear system and has led to a number of interesting results (see $[16,17])$. We recall the definitions of homogeneous systems with weighted dilation.

Definition 1. For real numbers $r_{i}>0, i=1, \ldots, n$ and fixed coordinates $\left(x_{1}, x_{2}, \ldots, x_{n}\right) \in \mathbb{R}^{n}, \forall \varepsilon>0$.

(i) The dilation $\Delta_{\varepsilon}(x)$ is defined by $\Delta_{\varepsilon}(x)=\left(\varepsilon^{r_{1}} x_{1}, \ldots\right.$, $\left.\varepsilon^{r_{n}} x_{n}\right), \forall \varepsilon>0$, with $r_{i}$ being called as the weights of the coordinates. For simplicity of notation, we define dilation weight $\Delta=\left(r_{1}, \ldots, r_{n}\right)$.

(ii) A function $V \in C\left(\mathbb{R}^{n}, \mathbb{R}\right)$ is said to be homogeneous of degree $\tau$ if there is a real number $\tau \in \mathbb{R}$ such that

$$
V\left(\Delta_{\varepsilon}(x)\right)=\varepsilon^{\tau} V\left(x_{1}, \ldots, x_{n}\right), \quad \forall x \in \mathbb{R}^{n} \backslash\{0\} .
$$

(iii) A vector field $f \in C\left(\mathbb{R}^{n}, \mathbb{R}^{n}\right)$ is said to be homogeneous of degree $\tau$ if there is a real number $\tau \in \mathbb{R}$ such that for $i=1, \ldots, n$

$$
f_{i}\left(\Delta_{\varepsilon}(x)\right)=\varepsilon^{\tau+r_{i}} f_{i}\left(x_{1}, \ldots, x_{n}\right), \quad \forall x \in \mathbb{R}^{n} \backslash\{0\} .
$$

(iv) A homogeneous $p$-norm is defined as $\|x\|_{\Delta, p}=$ $\left(\sum_{i=1}^{n}\left|x_{i}\right|^{p / r_{i}}\right)^{1 / p}, \forall x \in \mathbb{R}^{n}$, for a constant $p \geq 1$. For the simplicity, we choose $p=2$ and write $\|x\|_{\Delta}$ for $\|x\|_{\Delta, 2}$.
Lemma 2. Suppose $V: \mathbb{R}^{n} \rightarrow \mathbb{R}$ is a homogeneous function of degree $\tau$ with respect to the dilation weight $\Delta$. Then the following holds:

(i) $\partial V / \partial x_{i}$ is homogeneous of degree $\tau-r_{i}$ with $r_{i}$ being the homogeneous weight of $x_{i}$.

(ii) There is a constant $c$ such that

$$
V(x) \leq c\|x\|_{\Delta}^{\tau} .
$$

Moreover, if $V(x)$ is positive definite,

$$
\underline{c}\|x\|_{\Delta}^{\tau} \leq V(x), \text { for a constant } \underline{c}>0 .
$$

Lemma 3. For $x \in \mathbb{R}, y \in \mathbb{R}, p \geq 1$ is a constant; the following inequalities hold:

$$
\begin{aligned}
|x+y|^{p} & \leq 2^{p-1}\left|x^{p}+y^{p}\right|, \\
(|x|+|y|)^{1 / p} & \leq|x|^{1 / p}+|y|^{1 / p} \\
& \leq 2^{(p-1) / p}(|x|+|y|)^{1 / p} .
\end{aligned}
$$

If $p \geq 1$ is an odd integer or a ratio of two odd integers,

$$
\begin{aligned}
|x-y|^{p} & \leq 2^{p-1}\left|x^{p}-y^{p}\right| \\
\left|x^{p}-y^{p}\right| & \leq p|x-y|\left(x^{p-1}+y^{p-1}\right) \\
& \leq c|x-y|\left|(x-y)^{p-1}+y^{p-1}\right|,
\end{aligned}
$$

where $c>0$ is a constant.

Lemma 4. Suppose $c$ and $d$ are two positive real numbers. Given any positive number $\gamma>0$, the following inequality holds:

$$
|x|^{c}|y|^{d} \leq \frac{c}{c+d} \gamma|x|^{c+d}+\frac{d}{c+d} \gamma^{-c / d}|y|^{c+d} .
$$

Lemma 5. Let $0 \leq \delta_{1} \leq \cdots \leq \delta_{n}$ be real numbers and $c_{j} \geq$ $0, j=1, \ldots, n$. Then, $\forall x \in \mathbb{R}$,

$$
\begin{aligned}
c_{1}|x|^{\delta_{1}}+c_{n}|x|^{\delta_{n}} & \leq \sum_{j=1}^{n} c_{j}|x|^{\delta_{j}} \\
& \leq\left(\sum_{j=1}^{n} c_{j}\right)\left(|x|^{\delta_{1}}+|x|^{\delta_{n}}\right) .
\end{aligned}
$$

\section{Homogenous Stabilizer by State Feedback}

In this section, we first construct a set of Lyapunov functions for all subsystems of the following switched nonlinear systems:

$$
\begin{aligned}
& \dot{x}_{i}=x_{i+1}+f_{i, \sigma(t)}\left(\bar{x}_{i}\right), \quad i=1, \ldots, n-1 \\
& \dot{x}_{n}=u_{\sigma(t)}+f_{n, \sigma(t)}(x)
\end{aligned}
$$

which is exactly in (1) with $g_{i, \sigma(t)}(x)=0$. Then we present the homogeneous controllers of subsystems and a switching law for system (10) under some regular conditions. 
To the best of our knowledge, no results on switched nonlinear systems (10) in the use of the homogeneous domination approach have appeared. For system (10), a common Lyapunov function is difficult to find because a common coordinate transformation of all subsystems is difficult to be exploited. On the other hand, if system (1) has only one subsystem, the design problem reduces to the classical stabilization design problem for nonswitched nonlinear systems. According to the structure of system (1), however, system (1) cannot constructively be stabilized by the existing methods such as the adding a power integrator technique proposed in $[18,19]$. To obtain the homogenous state feedback controllers of subsystems and a switching law, which globally stabilize system (1), some assumptions are made as follows.

Assumption 6. For $i=1, \ldots, n$, and $k \in M$, there are constants $\tau_{k} \geq 0$ and $\rho_{k} \geq 0$, such that

$$
\begin{aligned}
& \left|f_{i, k}\left(\bar{x}_{i}\right)\right| \\
& \quad \leq \rho_{k}\left(\left|x_{1}\right|^{r_{i+1, k} / r_{1, k}}+\left|x_{2}\right|^{r_{i+1, k} / r_{2, k}}+\cdots+\left|x_{i}\right|^{r_{i+1, k} / r_{i, k}}\right)
\end{aligned}
$$

with the constants $r_{i, k}$ 's defined as

$$
\begin{aligned}
r_{1, k} & =1, \\
r_{i+1, k} & =r_{i, k}+\tau_{k} .
\end{aligned}
$$

Assumption 7. There are some constants $a_{l}, l=1, \ldots, k$ such that

$$
\begin{aligned}
& \left|g_{i, k}(x)\right| \\
& \leq a_{1}\left(\left|\xi_{1,1}\right|^{(n-1) \tau_{1} / r_{1, k}}+\cdots+\left|\xi_{i, 1}\right|^{(n-1) \tau_{1} / r_{1, k}}\right)+\cdots \\
& \quad+a_{k}\left(\left|\xi_{1, k}\right|^{(n-1) \tau_{k} / r_{i, k}}+\cdots+\left|\xi_{i, k}\right|^{(n-1) \tau_{k} / r_{i, k}}\right),
\end{aligned}
$$

where $\xi_{i, k}$ will be defined later.

Assumption 8. In the above formula, the variables must meet

$$
\tau_{i} \leq \min \left\{\tau_{1}, \ldots, \tau_{i-1}\right\}
$$

Remark 9. When $M=\{1\}$ and $g_{i, k}(x)=0$, Assumption 6 is reduced to that in $[12,14]$. In presence of $g_{i, k}(x)$, system (1) is more general compared with previous lower-triangular or upper-triangular system. If $\tau_{k}=0$, Assumptions 6 and 7 become linear growth conditions. Assumption 8 plays an important role in the later control design. For simplicity, in this paper we assume $\tau_{i}=q_{i} / p_{i}$ with $q_{i}$ an even integer and $p_{i}$ an odd integer. Based on this, $r_{i, j}=1+(j-1) \tau_{i}, j=1, \ldots, n+1$ will be odd in both denominator and numerator.

Theorem 10. Under Assumptions 6-8, there are constants $\delta_{i}, i=1, \ldots, n$ and continuous functions $\chi_{k, l}(x)>0, k, l \in$ $M$, such that for $\forall x \neq 0, k \in M$

$$
a \sum_{i=1}^{n} \delta_{i} \xi_{i, k}^{2 r_{n, k} / r_{i, k}}+\sum_{l=1}^{m} \chi_{k, l}(x)\left(V_{l}\left(\bar{\xi}_{n, l}\right)-V_{k}\left(\bar{\xi}_{n, k}\right)\right)<0 .
$$

Then, there exist homogeneous state feedback controllers and a switching law solving the global stabilization problem of system (1).

Proof. The proof is carried out by using an inductive argument, which enables us to simultaneously construct a Lyapunov function which is positive definite and proper, as well as homogeneous stabilizers. First construct a set of Lyapunov functions for all subsystems of system (10) using adding a power integrator technique for each $k$.

Step 1. Choose a Lyapunov function $V_{1, k}=\left(r_{1, k} /\left(2 r_{n, k}-\right.\right.$ $\left.\left.\tau_{k}\right)\right) x_{1}^{\left(2 r_{n, k}-\tau_{k}\right) / r_{1, k}}$. For each subsystem $k$ of switched system (10), the time derivative of $V_{1, k}$ along the trajectory of (10) is

$$
\begin{aligned}
\dot{V}_{1, k} & =x_{1}^{\left(\left(2 r_{n, k}-\tau_{k}\right) / r_{1, k}\right)-1}\left(x_{2}+f_{1, k}\left(x_{1}\right)\right) \\
& \leq x_{1}^{\left(\left(2 r_{n, k}-\tau_{k}\right) / r_{1, k}\right)-1}\left(x_{2}-x_{2, k}^{*}+x_{2, k}^{*}+x_{1}^{r_{2, k} / r_{1, k}} \rho_{k}\right) ;
\end{aligned}
$$

the last inequality is deduced by Assumption 6. Then choosing the virtual controller $x_{2, k}^{*}$ as

$$
x_{2, k}^{*}=-x_{1}^{r_{2, k} / r_{1, k}}\left(n+\rho_{k}\right):=-x_{1}^{r_{2, k} / r_{1, k}} \beta_{1, k}
$$

we can obtain

$$
\dot{V}_{1, k}\left(x_{1}\right) \leq x_{1}^{\left(\left(2 r_{n, k}-\tau_{k}\right) / r_{1, k}\right)-1}\left(x_{2}-x_{2, k}^{*}\right)-n x_{1}^{2 r_{n, k} / r_{1, k}} .
$$

Inductive Step. Suppose, at step $i-1$, there exists a Lyapunov function $V_{i-1, k}$, which is positive define and homogeneous with respect to (12) and a set of virtual controllers $x_{1, k}^{*}, \ldots, x_{i, k}^{*}$ defined by

$$
\begin{gathered}
x_{1, k}^{*}=0, \\
x_{2, k}^{*}=-\xi_{1, k}^{r_{2, k} / r_{1, k}} \beta_{1, k},
\end{gathered}
$$$$
x_{j, k}^{*}=-\xi_{j-1, k}^{r_{j, k} / r_{j-1, k}} \beta_{j-1, k},
$$$$
\xi_{1, k}=x_{1}-x_{1, k}^{*}
$$$$
\xi_{2, k}=x_{2}-x_{2, k}^{*}
$$

$$
\xi_{j, k}=x_{j}-x_{j, k}^{*}
$$

where $j=2, \ldots, i$ and $\beta_{1, k}>0, \ldots, \beta_{i-1, k}>0$ such that

$$
\begin{gathered}
\dot{V}_{i-1, k} \leq \xi_{i-1, k}^{\left(\left(2 r_{n, k}-\tau_{k}\right) / r_{i-1, k}\right)-1}\left(x_{i}-x_{i, k}^{*}\right) \\
-(n-i+2) \sum_{j=1}^{i-1} \xi_{j, k}^{2 r_{n, k} / r_{j, k}} .
\end{gathered}
$$

Next, we claim that (20) also holds at step $i$. To prove this claim, we consider the Lyapunov function

$$
V_{i, k}=V_{i-1, k}+\frac{r_{i, k}}{2 r_{n, k}-\tau_{k}} \xi_{i, k}^{\left(2 r_{n, k}-\tau_{k}\right) / r_{i, k}}
$$


whose derivative is

$$
\begin{aligned}
& \dot{V}_{i, k} \\
& \leq-(n-i+2) \sum_{j=1}^{i-1} \xi_{j, k}^{2 r_{n, k} / r_{j, k}}+\xi_{i-1, k}^{\left(\left(2 r_{n, k}-\tau_{k}\right) / r_{i-1, k}\right)-1} \xi_{i, k} \\
& \quad+\xi_{i, k}^{\left(\left(2 r_{n, k}-\tau_{k}\right) / r_{i, k}\right)-1}\left(x_{i+1}+f_{i, k}(\cdot)-\sum_{l=1}^{i-1} \frac{\partial x_{i, k}^{*}}{\partial x_{l}} \dot{x}_{l}\right) .
\end{aligned}
$$

Next, we estimate the last term on the right-hand side of (22). By Lemma 4, there are constants $c_{i, k}>0$ and $d_{i, k}>0$ such that

$$
\begin{aligned}
\xi_{i-1, k}^{\left(\left(2 r_{n, k}-\tau_{k}\right) / r_{i-1, k}\right)-1} \xi_{i, k} & =\xi_{i-1, k}^{\left(2 r_{n, k}-\tau_{k}-r_{i-1, k}\right) / r_{i-1, k}} \xi_{i, k}^{\left(1 / r_{i, k}\right) r_{i, k}} \\
& \leq c_{i, k} \xi_{i-1, k}^{2 r_{n, k} / r_{i-1, k}}+d_{i, k} \xi_{i, k}^{2 r_{n, k} / r_{i, k}} .
\end{aligned}
$$

By Lemma 3, there is a constant $\tilde{\rho}_{k}$ such that Assumption 6 can be rewritten as

$$
\begin{aligned}
& \left|f_{i, k}\left(\bar{x}_{i}\right)\right| \\
& \quad \leq \rho_{k}\left[\left|x_{1}\right|^{r_{i+1, k} / r_{1, k}}+\left|x_{2}\right|^{r_{i+1, k} / r_{2, k}}+\cdots+\left|x_{i}\right|^{r_{i+1, k} / r_{i, k}}\right] \\
& \quad=\rho_{k} \sum_{j=1}^{i}\left|\xi_{j, k}-\xi_{j-1, k}^{r_{j, k} / r_{j-1, k}} \beta_{j-1, k}\right|^{\left(r_{i, k}+\tau_{k}\right) / r_{j, k}} \\
& \quad \leq \tilde{\rho}_{k} \sum_{j=1}^{i}\left|\xi_{j, k}\right|^{\left(r_{i, k}+\tau_{k}\right) / r_{j, k}} .
\end{aligned}
$$

On the other hand, one has

$\left|\sum_{l=1}^{i-1} \frac{\partial x_{i, k}^{*}}{\partial x_{l}} \dot{x}_{l}\right|=\sum_{l=1}^{i-1} \frac{\partial\left(-\xi_{i-1, k}^{\tau_{k} / r_{i-1, k}} \beta_{i-1, k}\right)}{\partial x_{l}}\left(x_{l+1}+f_{l, k}(\cdot)\right)$.

According to definition (19), one obtains

$$
\frac{\partial x_{i, k}^{*}}{\partial x_{l}}=\frac{\partial\left(-\xi_{i-1, k}^{\tau_{k} / r_{i-1, k}} \beta_{i-1, k}\right)}{\partial x_{l}} \leq m \xi_{i-1, k}^{\tau_{k} / r_{i-1, k}} \cdots \xi_{l, k}^{\tau_{k} / r_{l, k}}
$$

Combining (26) and (24) into (25), we have

$$
\begin{aligned}
& \left|\sum_{l=1}^{i-1} \frac{\partial x_{i, k}^{*}}{\partial x_{l}} \dot{x}_{l}\right| \leq \sum_{l=1}^{i-1} m\left|\xi_{i-1, k}^{\tau_{k} / r_{i-1, k}} \ldots \xi_{l, k}^{\tau_{k} / r_{l, k}}\right| \\
& \cdot\left(\xi_{l+1, k}-\xi_{l, k}^{r_{l+1, k} / r_{l, k}} \beta_{l, k}+\widetilde{\rho}_{k} \sum_{j=1}^{i}\left|\xi_{j, k}\right|^{\left(r_{i, k}+\tau_{k}\right) / r_{j, k}}\right) .
\end{aligned}
$$

By Lemma 5 and the fact that $(i-l) \tau_{k}+r_{l, k}=r_{i, k}$, (27) becomes

$$
\begin{aligned}
& \left|\sum_{l=1}^{i-1} \frac{\partial x_{i, k}^{*}}{\partial x_{l}} \dot{x}_{l}\right| \leq \sum_{l=1}^{i-1} \widetilde{m} \sum_{j=1}^{l+1}\left|\xi_{j, k}\right|^{\left(\tau_{k}+r_{i, k}\right) / r_{j, k}} \\
& \quad \leq \bar{m}\left(\left|\xi_{1, k}\right|^{\left(\tau_{k}+r_{i, k}\right) / r_{1, k}}+\cdots+\left|\xi_{i, k}\right|^{\left(\tau_{k}+r_{i, k}\right) / r_{i, k}}\right),
\end{aligned}
$$

where $\widetilde{m}$ and $\bar{m}$ are positive constants.
Combining (23), (24), and (28) into (22) yields

$$
\begin{aligned}
& \dot{V}_{i, k} \leq(n-i+2) \sum_{j=1}^{i-1} \xi_{j, k}^{2 r_{n, k} / r_{j, k}}+c_{i, k} \xi_{i-1, k}^{2 r_{n, k} / r_{i-1, k}} \\
& +d_{i, k} \xi_{i, k}^{2 r_{n, k} / r_{i, k}}+\xi_{i, k}^{\left(\left(2 r_{n, k}-\tau_{k}\right) / r_{i, k}\right)-1} x_{i+1} \\
& +\xi_{i, k}^{\left(\left(2 r_{n, k}-\tau_{k}\right) / r_{i, k}\right)-1}\left(f_{i, k}(\cdot)-\sum_{l=1}^{i-1} \frac{\partial x_{i, k}^{*}}{\partial x_{l}} \dot{x}_{l}\right) \\
& \leq \xi_{i, k}^{\left(\left(2 r_{n, k}-\tau_{k}\right) / r_{i, k}\right)-1}\left(\widetilde{\rho}_{k} \sum_{j=1}^{i}\left|\xi_{j, k}\right|^{\left(r_{i, k}+\tau_{k}\right) / r_{j, k}}\right. \\
& \left.-\bar{m}\left(\left|\xi_{1, k}\right|^{\left(\tau_{k}+r_{i, k}\right) / r_{1, k}}+\cdots+\left|\xi_{i, k}\right|^{\left(\tau_{k}+r_{i, k}\right) / r_{i, k}}\right)\right)-(n \\
& -i+2) \sum_{j=1}^{i-1} \xi_{j, k}^{2 r_{n, k} / r_{j, k}}+c_{i, k} \xi_{i-1, k}^{2 r_{n, k} / r_{i-1, k}}+d_{i, k} \xi_{i, k}^{2 r_{n, k} / r_{i, k}} \\
& +\xi_{i, k}^{\left(\left(2 r_{n, k}-\tau_{k}\right) / r_{i, k}\right)-1} x_{i+1} \leq-(n-i+2) \sum_{j=1}^{i-1} \xi_{j, k}^{2 r_{n, k} / r_{j, k}} \\
& +c_{i, k} \xi_{i-1, k}^{2 r_{n, k} / r_{i-1, k}}+d_{i, k} \xi_{i, k}^{2 r_{n, k} / r_{i, k}}+\xi_{i, k}^{\left(\left(2 r_{n, k}-\tau_{k}\right) / r_{i, k}\right)-1}\left(\widetilde{\rho}_{k}\right. \\
& +\bar{m}) \sum_{j=1}^{i}\left|\xi_{j, k}\right|^{\left(r_{i, k}+\tau\right) / r_{j, k}}+\xi_{i, k}^{\left(\left(2 r_{n, k}-\tau_{k}\right) / r_{i, k}\right)-1} x_{i+1} .
\end{aligned}
$$

Choosing appropriate constants $c_{i, k}, d_{i, k}, m_{i, k}$, and $n_{i, k}$, (29) becomes

$$
\begin{aligned}
\dot{V}_{i, k} \leq & -(n-i+1) \sum_{j=1}^{i} \xi_{j, k}^{2 r_{n, k} / r_{j, k}}+\xi_{i, k}^{\left(\left(2 r_{n, k}-\tau_{k}\right) / r_{i, k}\right)-1} \\
& \times\left(x_{i+1}+\left(d_{i, k}+n_{i, k}\right) \xi_{i, k}^{r_{i+1, k} / r_{i, k}}\right) .
\end{aligned}
$$

Next, we construct controllers for each subsystem and a switching law which meet the requirements of the MLFs framework for system (1). We can choose the Lyapunov function $V_{k}=(1 / 2) \xi_{1, k}^{2}+\sum_{i=2}^{n}\left(r_{i, k} /\left(2 r_{n, k}-\tau_{k}\right)\right) \xi_{i, k}^{\left(2 r_{n, k}-\tau_{k}\right) / r_{i, k}}$. From (16)-(30), it is not hard to arrive at

$$
\begin{aligned}
\dot{V}_{k}= & x_{1}\left(x_{2}+f_{1, k}\left(x_{1}\right)+g_{1, k}(x)\right)+\sum_{i=2}^{n-1} \xi_{i, k}^{\left(\left(2 r_{n, k}-\tau_{k}\right) / r_{i, k}\right)-1} \\
& \times\left(x_{i+1}+f_{i, k}\left(\bar{x}_{i}\right)+g_{i, k}(x)-\sum_{l=1}^{i-1} \frac{\partial x_{i, k}^{*}}{\partial x_{l}} \dot{x}_{l}\right) \\
& +\xi_{n, k}^{\left(\left(2 r_{n, k}-\tau_{k}\right) / r_{i, k}\right)-1}\left(u_{k}+f_{n, k}(x)-\sum_{l=1}^{n-1} \frac{\partial x_{n, k}^{*}}{\partial x_{l}} \dot{x}_{l}\right) .
\end{aligned}
$$

Using inequalities (26), (27), (28), and (30), one has

$$
\begin{aligned}
\dot{V}_{k} & \leq-2 \sum_{j=1}^{n-1} \xi_{j, k}^{2 r_{n, k} / r_{j, k}}+\xi_{n-1, k}^{\left(\left(2 r_{n, k}-\tau_{k}\right) / r_{n-1, k}\right)-1}\left(x_{n}\right. \\
& \left.+p \xi_{n-1, k}^{r_{n, k} / r_{n-1, k}}\right)+x_{1} g_{1, k}(x)
\end{aligned}
$$




$$
\begin{aligned}
& +\sum_{i=2}^{n-1} \xi_{i, k}^{\left(\left(2 r_{n, k}-\tau_{k}\right) / r_{i, k}\right)-1}\left(g_{i, k}(x)-\sum_{l=1}^{i-1} \frac{\partial x_{i, k}^{*}}{\partial x_{l}} g_{l, k}(x)\right) \\
& +\xi_{n, k}^{\left(\left(2 r_{n, k}-\tau_{k}\right) / r_{n, k}\right)-1}\left(u_{k}+f_{n, k}(x)-\sum_{l=1}^{n-1} m_{1} \xi_{n-1, k}^{\tau_{k} / r_{n-1, k}}\right. \\
& \ldots \xi_{l, k}^{\tau_{k} / r_{l, k}}\left(\xi_{l+1, k}-\xi_{l, k}^{r_{l+1, k} / r_{l, k}} \beta_{l, k}\right. \\
& \left.\left.+\widetilde{\rho}_{k} \sum_{j=1}^{l}\left|\xi_{j, k}\right|^{\left(r_{l, k}+\tau_{k}\right) / r_{j, k}}+g_{l, k}(x)\right)\right)
\end{aligned}
$$

where $p, m_{1}$, and $\widetilde{\rho}_{k}$ are positive constants.

According to Lemma 4 and (19), there exist positive constants $p, p_{1}$, and $p_{2}$ such that

$$
\begin{aligned}
& \xi_{n-1, k}^{\left(\left(2 r_{n, k}-\tau_{k}\right) / r_{n-1, k}\right)-1}\left(x_{n}+p \xi_{n-1, k}^{r_{n, k} / r_{n-1, k}}\right) \\
& \quad \leq p \xi_{n-1, k}^{2 r_{n, k} / r_{n-1, k}}+\xi_{n-1, k}^{r_{n, k} / r_{n-1, k}}\left(\xi_{n, k}-\beta_{n-1, k} \xi_{n-1, k}^{r_{n, k} / r_{n-1, k}}\right) \\
& \quad \leq p_{1} \xi_{n-1, k}^{2 r_{n, k} / r_{n-1, k}}+p_{2} \xi_{n, k}^{2 r_{n, k} / r_{n, k}} .
\end{aligned}
$$

By Lemma 4 and (24) and (28), there exists a constant $\alpha_{i}$ such that

$$
\begin{aligned}
& \mid \xi_{n, k}^{\left(\left(2 r_{n, k}-\tau_{k}\right) / r_{n, k}\right)-1}\left(f_{n, k}(\bar{x})-\sum_{l=1}^{n-1} m_{1} \xi_{n-1, k}^{\tau_{k} / r_{n-1, k}}\right. \\
& \quad \ldots \xi_{l, k}^{\tau_{k} / r_{l, k}}\left(\xi_{l+1, k}-\xi_{l, k}^{r_{l+1, k} / r_{l, k}} \beta_{l, k}\right. \\
& \left.\left.+\tilde{\rho}_{k} \sum_{j=1}^{l}\left|\xi_{j, k}\right|^{\left(r_{n, k}+\tau\right) / r_{j, k}}\right)\right) \mid \\
& \leq \mid \xi_{n, k}^{\left(\left(2 r_{n, k}-\tau_{k}\right) / r_{n, k}\right)-1}\left(\rho_{n} \sum_{j=1}^{n}\left|\xi_{j, k}\right|^{\left(r_{n, k}+\tau\right) / r_{j, k}}\right.
\end{aligned}
$$

$$
\begin{aligned}
& \left.-m_{2}\left(\left|\xi_{1, k}\right|^{\left(\tau_{k}+r_{n, k}\right) / r_{1, k}}+\cdots+\left|\xi_{n, k}\right|^{\left(\tau_{k}+r_{n, k}\right) / r_{n, k}}\right)\right) \mid \\
& \leq \alpha_{i} \sum_{i=1}^{n}\left|\xi_{i, k}\right|^{2 r_{n, k} / r_{i, k}}
\end{aligned}
$$

Combining (33) and (34) into (32), we obtain $\dot{V}_{k}$

$$
\begin{aligned}
\leq & -2 \sum_{j=1}^{n-1} \xi_{j, k}^{2 r_{n, k} / r_{j, k}}+p_{1} \xi_{n-1, k}^{2 r_{n, k} / r_{n-1, k}}+p_{2} \xi_{n, k}^{2 r_{n, k} / r_{n, k}} \\
& +\xi_{n-1, k}^{\left(\left(2 r_{n, k}-\tau_{k}\right) / r_{n-1, k}\right)-1} x_{1} g_{1, k}(x) \\
& +\sum_{i=2}^{n-1} \xi_{i, k}^{\left(\left(2 r_{n, k}-\tau_{k}\right) / r_{i, k}\right)-1}\left(g_{i, k}(x)-\sum_{l=1}^{i-1} \frac{\partial x_{i, k}^{*}}{\partial x_{l}} g_{l, k}(x)\right) \\
& +\xi_{n, k}^{\left(\left(2 r_{n, k}-\tau_{k}\right) / r_{n, k}\right)-1} u_{k}+\sum_{i=1}^{n} \alpha_{i}\left|\xi_{i, k}\right|^{2 r_{n, k} / r_{i, k}} \\
& -\xi_{n, k}^{\left(\left(2 r_{n, k}-\tau_{k}\right) / r_{n, k}\right)-1} \sum_{l=1}^{n-1} m_{1} \xi_{n-1, k}^{\tau_{k} / r_{n-1, k}} \ldots \xi_{l, k}^{\tau_{k} / r_{l, k}} g_{l, k}(x) .
\end{aligned}
$$

After the merger, we have

$\dot{V}_{k}$

$$
\begin{aligned}
\leq & -2 \sum_{j=1}^{n-1} \xi_{j, k}^{2 r_{n, k} / r_{j, k}}+p_{1} \xi_{n-1, k}^{2 r_{n, k} / r_{n-1, k}}+p_{2} \xi_{n, k}^{2 r_{n, k} / r_{n, k}} \\
& +\xi_{n, k}^{\left(\left(2 r_{n, k}-\tau_{k}\right) / r_{n, k}\right)-1} u_{k}+\alpha_{i} \sum_{i=1}^{n}\left|\xi_{i, k}\right|^{2 r_{n, k} / r_{i, k}} \\
& +\xi_{n-1, k}^{\left(\left(2 r_{n, k}-\tau_{k}\right) / r_{n-1, k}\right)-1} \sum_{i=2}^{n-1} \xi_{i, k}^{\left(\left(2 r_{n, k}-\tau_{k}\right) / r_{i, k}\right)-1} g_{i, k}(x) \\
& +m_{2} \sum_{i=2}^{n} \sum_{l=1}^{i-1} \xi_{i, k}^{\left(\left(2 r_{n, k}-\tau_{k}\right) / r_{i, k}\right)-1} \xi_{i-1, k}^{\tau_{k} / r_{i-1, k}} \ldots \xi_{l, k}^{\tau_{k} / r_{l, k}} g_{l, k}(x) .
\end{aligned}
$$

According to Assumptions 7 and 8, one has

$$
\begin{aligned}
& \left|\xi_{n-1, k}^{\left(\left(2 r_{n, k}-\tau_{k}\right) / r_{n-1, k}\right)-1} \sum_{i=2}^{n-1} \xi_{i, k}^{\left(\left(2 r_{n, k}-\tau_{k}\right) / r_{i, k}\right)-1} g_{i, k}(x)+m_{2} \sum_{i=2}^{n} \sum_{l=1}^{i-1} \xi_{i, k}^{\left(\left(2 r_{n, k}-\tau_{k}\right) / r_{i, k}\right)-1} \xi_{i-1, k}^{\tau_{k} / r_{i-1, k}} \cdots \xi_{l, k}^{\tau_{k} / r_{l, k}} g_{l, k}(x)\right| \\
& \quad \leq \mid \xi_{n-1, k}^{\left(\left(2 r_{n, k}-\tau_{k}\right) / r_{n-1, k}\right)-1} \sum_{i=2}^{n-1} \xi_{i, k}^{\left(\left(2 r_{n, k}-\tau_{k}\right) / r_{i, k}\right)-1}\left(a_{1}\left(\left|\xi_{1,1}\right|^{(n-1) \tau_{1} / r_{1, k}}+\cdots+\left|\xi_{i, 1}\right|^{(n-1) \tau_{1} / r_{1, k}}\right)+\cdots\right. \\
& \left.\quad+a_{k}\left(\left|\xi_{1, k}\right|^{(n-1) \tau_{k} / r_{i, k}}+\cdots+\left|\xi_{i, k}\right|^{(n-1) \tau_{k} / r_{i, k}}\right)\right) \mid+m_{2} \sum_{i=2}^{n} \sum_{l=1}^{i-1} \xi_{i, k}^{\left(\left(2 r_{n, k}-\tau_{k}\right) / r_{i, k}\right)-1} \xi_{i-1, k}^{\tau_{k} / r_{i-1, k}} \ldots \xi_{l, k}^{\tau_{k} / r_{l, k}}\left(a _ { 1 } \left(\left|\xi_{1,1}\right|^{(n-1) \tau_{1} / r_{1, k}}+\cdots\right.\right. \\
& \left.\left.\quad+\left|\xi_{l, 1}\right|^{(n-1) \tau_{1} / r_{1, k}}\right)+\cdots+a_{k}\left(\left|\xi_{1, k}\right|^{(n-1) \tau_{k} / r_{i, k}}+\cdots+\left|\xi_{l, k}\right|^{(n-1) \tau_{k} / r_{i, k}}\right)\right) \leq d_{i} \sum_{j=1}^{n} \xi_{j, k}^{2 r_{n, k} / r_{j, k}} .
\end{aligned}
$$


Choose the state feedback controllers $u_{k}, k \in M$ and a reasonable function $q_{k}(x)$ as

$$
u_{k}=-\xi_{n, k}^{\left(r_{n, k}+\tau_{k}\right) / r_{n, k}} q_{k}(x)
$$

By appropriate choice, we have

$$
\dot{V}_{k} \leq \sum_{i=1}^{n} \delta_{i} \xi_{i, k}^{2 r_{n, k} / r_{i, k}} \leq-\sum_{l=1}^{m} \chi_{k l}(x)\left(V_{l}\left(\bar{\xi}_{n l}\right)-V_{k}\left(\bar{\xi}_{n k}\right)\right),
$$

$\forall x \neq 0$,

where $V_{k}$ is a positive definite and proper Lyapunov function. Now we design the switching law as follows:

$$
\sigma(t)=\underset{k \in M}{\arg \min }\left\{V_{k}\left(\xi_{1, k}, \ldots, \xi_{n, k}\right)\right\}
$$

By inequalities (15), (39), and (40), when $\sigma(t)=k$, one has $\dot{V}_{k}<0, \forall x \neq 0$. As a result, according to the MLFs method, the closed-loop system (1) and (38) under the switching law (40) is globally asymptotically stable.

\section{An Illustrative Example}

In this section, we give an example to demonstrate the validity of the proposed method. Consider the switched nonlinear system as shown in [13]

$$
\begin{aligned}
& \dot{x}_{1}=x_{2} \\
& \dot{x}_{2}=x_{3}+g_{\sigma(t)}(x) \\
& \dot{x}_{3}=x_{4} \\
& \dot{x}_{4}=u_{\sigma(t)}+\left(\frac{m_{2} g r}{J_{2}}-\frac{k r^{2}}{4 J_{2}}\right) \sin x_{3}+\frac{k r^{2}}{4 J_{2}} \sin x_{2},
\end{aligned}
$$

where $\sigma(t)=\{1,2\}, m_{2}=2.5 \mathrm{~kg}, g=9.81 \mathrm{~m} / \mathrm{s}^{2}, r=0.5 \mathrm{~m}$, and $J_{2}=0.625 \mathrm{~kg} \cdot g_{1}(x)=-(1 / 32) x_{4}-(1 / 64) x_{3}^{2}-(1 / 16) x_{2} x_{3}^{2}-$ $(5 / 64) x_{1} x_{3}^{2}-(1 / 4) x_{3}-(13 / 16) x_{2}-(27 / 32) x_{1}$ and $g_{2}(x)=$ $-(1 / 36) x_{4}-(1 / 36) x_{3}^{2}-(1 / 9) x_{2} x_{3}^{2}-(5 / 36) x_{1} x_{3}^{2}-(7 / 36) x_{3}-$ $(11 / 18) x_{2}-(11 / 18) x_{1}$. Following the design in Section 3, choosing the MLFs functions $V_{k}=(1 / 2) \xi_{1}^{2}+(1 / 2) \xi_{2}^{2}+$ $(1 / 2) \xi_{3}^{2}+(1 / 2) \xi_{4 k}^{2}$, where $\xi_{1}=x_{1}, \xi_{2}=x_{2}+2 x_{1}, \xi_{3}=$ $x_{3}+4 x_{2}+5 x_{1}, \xi_{4,1}=x_{4}+5 x_{3}+14 x_{2}+12 x_{1}+\left(2+x_{3}^{2}\right) \xi_{3}$, and $\xi_{4,1}=x_{4}+5 x_{3}+14 x_{2}+12 x_{1}+\left(3+0.5 x_{3}^{2}\right) \xi_{3}$, we design the controllers $u_{1}=-\xi_{3}-2 \xi_{41}-9.6 \sin x_{3}-10 \sin x_{2}-5 x_{4}-$ $14\left(x_{3}+g_{1}(x)\right)-12 x_{2}-2 x_{3} x_{4} \xi_{3}\left(2+x_{3}^{2}\right)\left(x_{4}+4 x_{3}+5 x_{2}+4 g_{1}(x)\right)$ and $u_{2}=-\xi_{3}-2 \xi_{42}-9.6 \sin x_{3}-10 \sin x_{2}-5 x_{4}-14\left(x_{3}+\right.$ $\left.g_{2}(x)\right)-12 x_{2}-2 x_{3} x_{4} \xi_{3}\left(3+0.5 x_{3}^{2}\right)\left(x_{4}+4 x_{3}+5 x_{2}+4 g_{2}(x)\right)$.

In the simulation, Figures 1-3 illustrate the effectiveness of control design under the switching signal $\sigma(t)=$ $\arg \min _{k=1,2}\left\{V_{k}\right\}$ with the initial condition $\left(x_{1}(0), x_{2}(0)\right.$, $\left.x_{3}(0), x_{4}(0)\right)^{T}=(-2,2,0.3,-1)^{T}$.

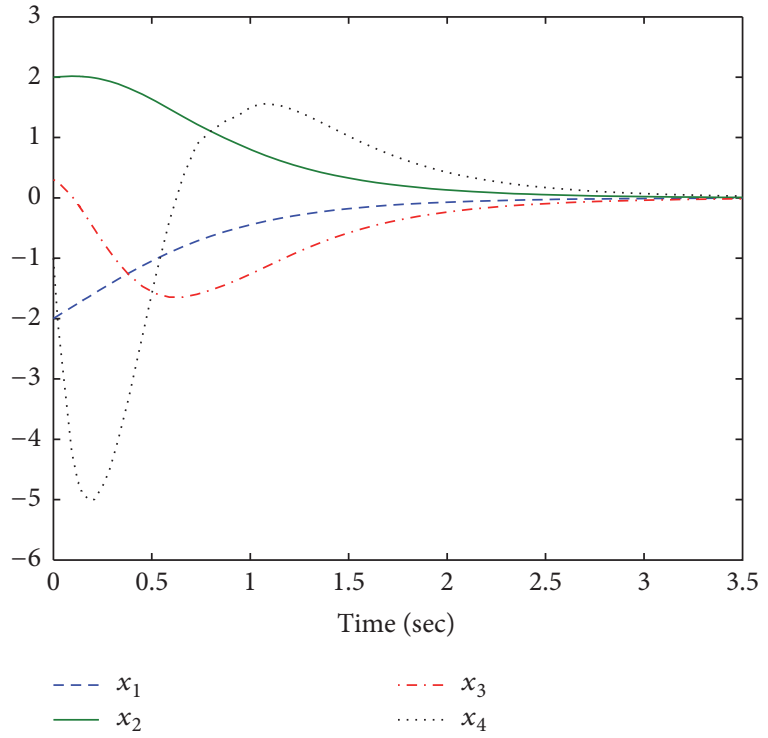

Figure 1: The curves of states $x_{1}, x_{2}, x_{3}$, and $x_{4}$.

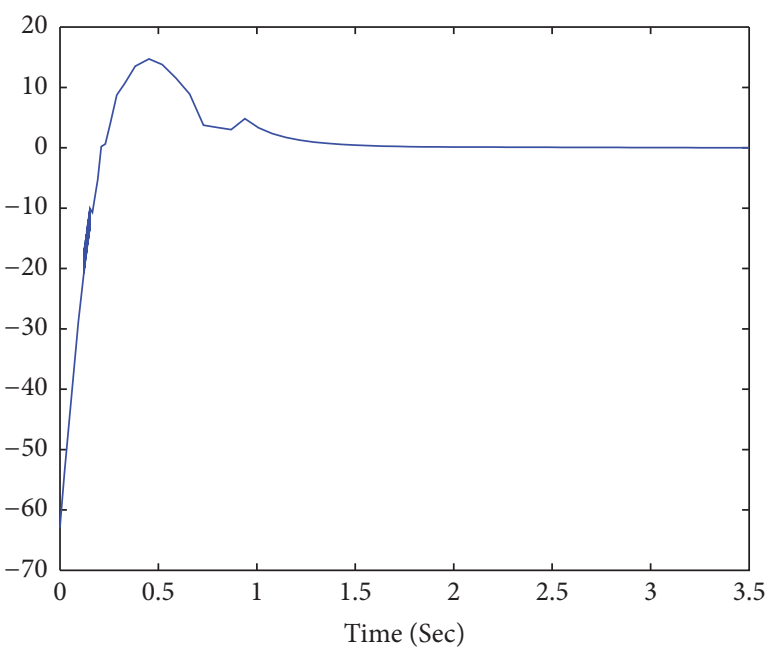

Figure 2: The curve of the control input $u$.

\section{Conclusion}

This paper has discussed the problem of global state feedback stabilization for a class of switched nonlinear systems. We design homogeneous controllers based on adding a power integrator technique and a switching law to guarantee asymptotical stability of the closed-loop switched nonlinear system.

\section{Conflicts of Interest}

The authors declare that they have no conflicts of interest. 


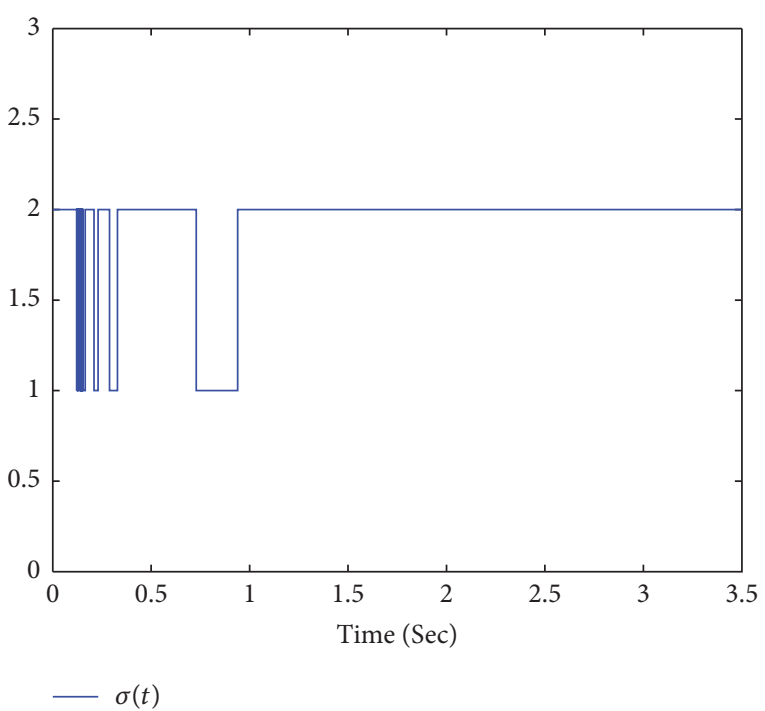

FIgURE 3: The switching signal $\sigma(t)$.

\section{Acknowledgments}

This work is supported by the National Natural Science Foundation of China (61773201, 61473143, and 61622304), Natural Science Foundation of Jiangsu province (BK20160035), and Fundamental Research Funds for the Central Universities (NE2014202, NE2015002).

\section{References}

[1] M. S. Branicky, "Multiple Lyapunov functions and other analysis tools for switched and hybrid systems," Institute of Electrical and Electronics Engineers Transactions on Automatic Control, vol. 43, no. 4, pp. 475-482, 1998.

[2] J.-y. Zhai, B. Wang, and S.-m. Fei, "Tracking control for switched nonlinear systems with multiple time-varying delays," Nonlinear Analysis: Hybrid Systems, vol. 17, pp. 44-55, 2015.

[3] W. Feng and J. Zhang, "Input-to-state stability of switched nonlinear systems," Science China Information Sciences, vol. 51, no. 12, pp. 1992-2004, 2008.

[4] N. H. El-Farra, P. Mhaskar, and P. D. Christofides, "Output feedback control of switched nonlinear systems using multiple Lyapunov functions," Systems and Control Letters, vol. 54, no. 12, pp. 1163-1182, 2005.

[5] L. Long and J. Zhao, " $\mathrm{H}_{\infty}$ control of switched nonlinear systems in $p$-normal form using multiple Lyapunov functions," Institute of Electrical and Electronics Engineers Transactions on Automatic Control, vol. 57, no. 5, pp. 1285-1291, 2012.

[6] J. Zhai and Z. Song, "Global finite-time stabilization for a class of switched nonlinear systems via output feedback," International Journal of Control, Automation, and Systems, vol. 15, no. 5, pp. 1975-1982, 2017.

[7] L. Long and J. Zhao, "Global stabilization for a class of switched nonlinear feedforward systems," Systems and Control Letters, vol. 60, no. 9, pp. 734-738, 2011.

[8] R. Ma and J. Zhao, "Backstepping design for global stabilization of switched nonlinear systems in lower triangular form under arbitrary switchings," Automatica, vol. 46, no. 11, pp. 1819-1823, 2010.
[9] J. Zhai, Z. Song, S. Fei, and Z. Zhu, "Global finte-time output feedback stabilisation for a class of switched high-order nonlinear systems," International Journal of Control, pp. 1-11, 2017.

[10] J.-L. Wu, "Stabilizing controllers design for switched nonlinear systems in strict-feedback form," Automatica, vol. 45, no. 4, pp. 1092-1096, 2009.

[11] C. Qian and W. Lin, "Output feedback control of a class of nonlinear systems: a nonseparation principle paradigm," Institute of Electrical and Electronics Engineers Transactions on Automatic Control, vol. 47, no. 10, pp. 1710-1715, 2002.

[12] C. Qian, "A homogeneous domination approach for global output feedback stabilization of a class of nonlinear systems," in Proceedings of the American Control Conference, pp. 4708-4715, Portland, Ore, USA, June 2005.

[13] L. Long and J. Zhao, "Global stabilization of switched nonlinear systems in non-triangular form and its application," Journal of The Franklin Institute, vol. 351, no. 2, pp. 1161-1178, 2014.

[14] Q. Gong and C. Qian, "Global practical tracking of a class of nonlinear systems by output feedback," Automatica, vol. 43, no. 1, pp. 184-189, 2007.

[15] D. Liberzon, Switching in Systems and Control, Birkhauser, Massachusetts, Mass, USA, 2003.

[16] H. Hermes, "Homogeneous coordinates and continuous asymptotically stabilizing feedback controls," in Differential Equations, vol. 127 of Lecture Notes in Pure and Appl. Math., pp. 249-260, Dekker, New York, NY, USA, 1991.

[17] M. Kawski, "Homogeneous stabilizing feedback laws," Control Theory and Advanced Technology, vol. 6, no. 4, pp. 497-516, 1990.

[18] W. Lin and C. Qian, "Adding a power integrator: a tool for global stabilization of high-order lower-triangular systems," in Proceeding of the 38th IEEE Conference on Decision and Control, pp. 1202-1207, Phoenix, Arizona, Ariz, USA, December 1999.

[19] C. Qian and W. Lin, "Non-Lipschitz continuous stabilizers for nonlinear systems with uncontrollable unstable linearization," Systems and Control Letters, vol. 42, no. 3, pp. 185-200, 2001. 


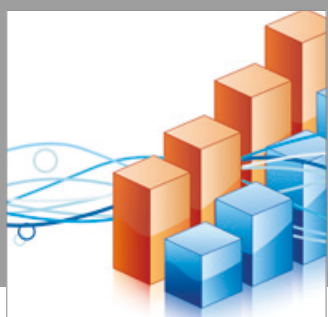

Advances in

Operations Research

vatersals

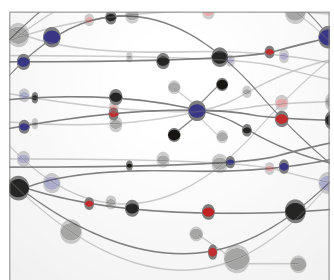

\section{The Scientific} World Journal
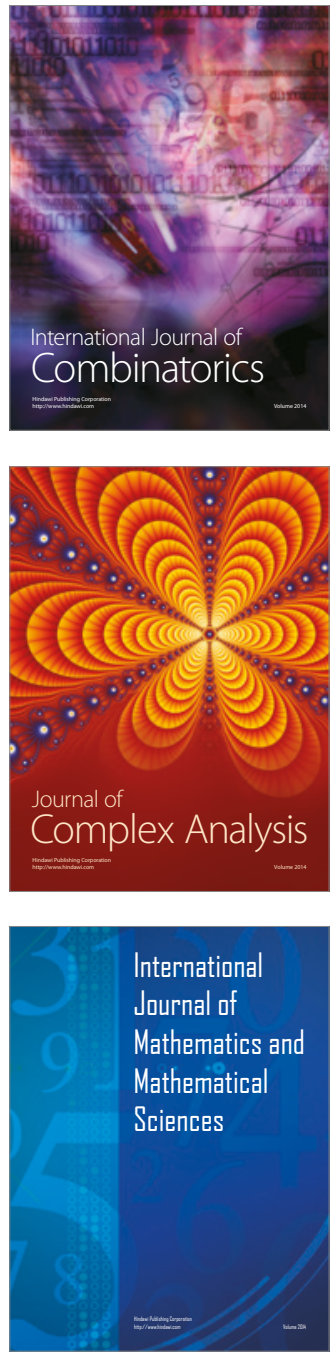
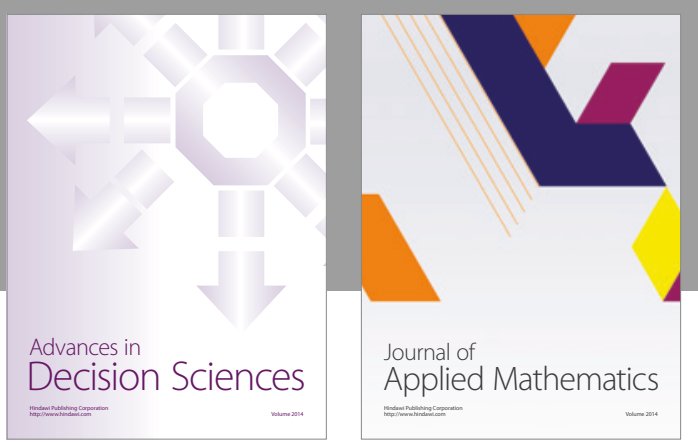

Algebra

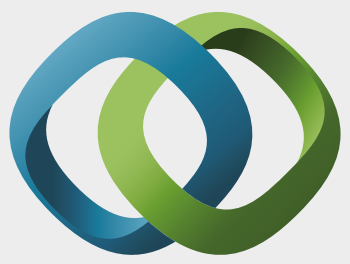

\section{Hindawi}

Submit your manuscripts at

https://www.hindawi.com
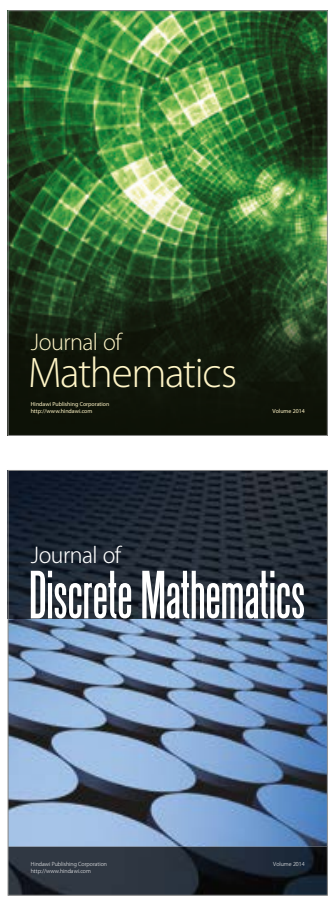

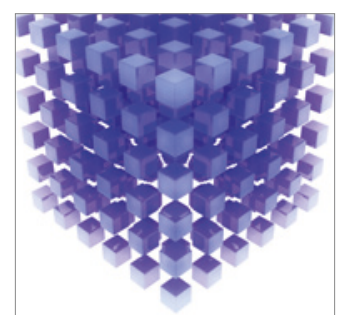

Mathematical Problems in Engineering
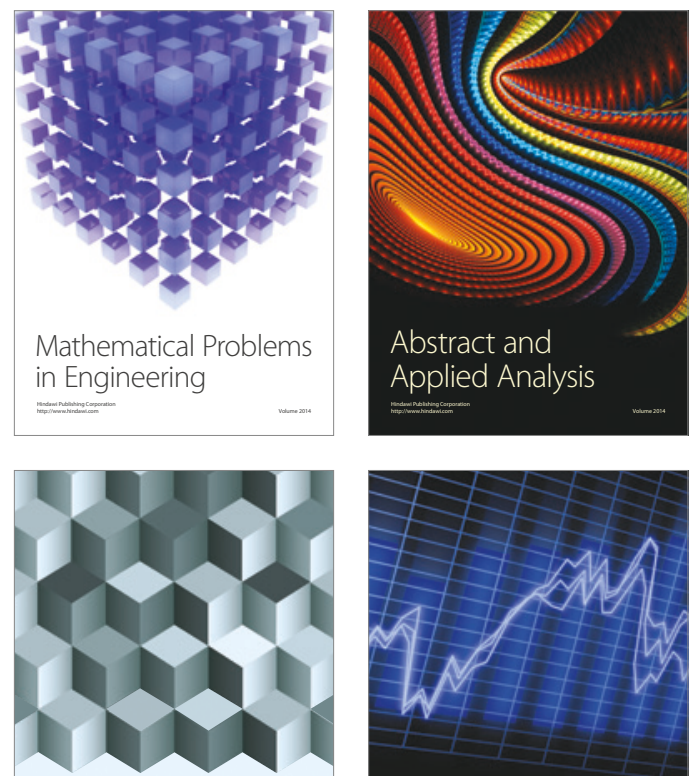

Journal of

Function Spaces

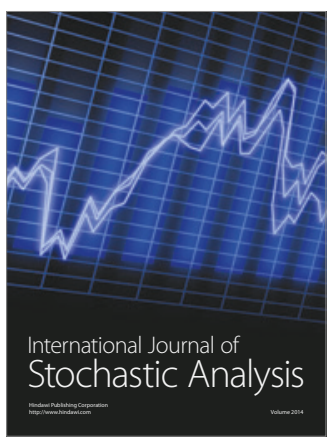

Probability and Statistics
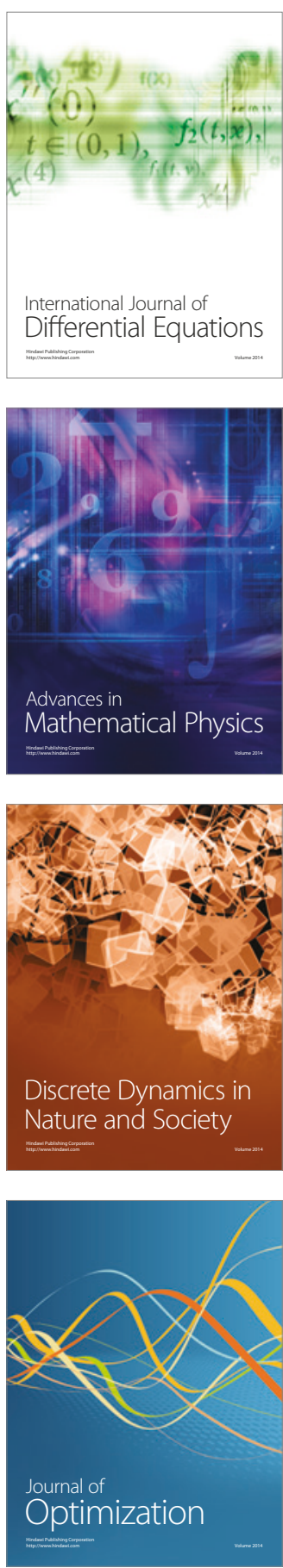